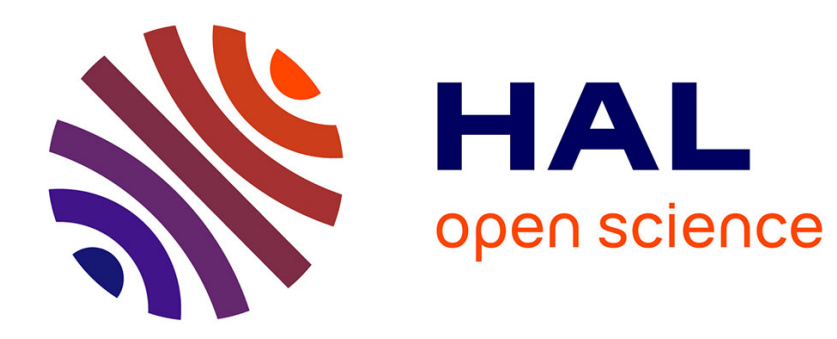

\title{
Conditions of Nucleation and Interface Propagation in Thermoplastic Materials
}

\author{
V. Levitas
}

\section{To cite this version:}

V. Levitas. Conditions of Nucleation and Interface Propagation in Thermoplastic Materials. Journal de Physique IV Proceedings, 1995, 05 (C8), pp.C8-173-C8-178. 10.1051/jp4:1995822 . jpa-00254071

\section{HAL Id: jpa-00254071 https://hal.science/jpa-00254071}

Submitted on 1 Jan 1995

HAL is a multi-disciplinary open access archive for the deposit and dissemination of scientific research documents, whether they are published or not. The documents may come from teaching and research institutions in France or abroad, or from public or private research centers.
L'archive ouverte pluridisciplinaire $\mathbf{H A L}$, est destinée au dépôt et à la diffusion de documents scientifiques de niveau recherche, publiés ou non, émanant des établissements d'enseignement et de recherche français ou étrangers, des laboratoires publics ou privés. 


\title{
Conditions of Nucleation and Interface Propagation in Thermoplastic Materials
}

\author{
V.I. Levitas \\ Institut für Baumechanik und Numerische Mechanik, Universität Hannover, Appelstrasse 9A, \\ 30167 Hannover, Germany
}

\begin{abstract}
Nucleation and interface propagation criteria are derived with account for temperature variation in the course of phase transition (PT) and internal variables. A model problem concerning the nucleation of thin inclined infinite layer in a rigid-plastic half-space under prescribed normal and shear stresses is solved. Nontrivial effect of shear stress on the PT pressure is shown.
\end{abstract}

\section{INTRODUCTION}

A lot of experiments exhibit very strong effect of plastic straining on martensitic PT, but corresponding theory makes the first steps only. The review of various approaches published before 1992 can be found in [1]. In [2,3] conditions of nucleation and interface propagation are derived. In the present paper these conditions are generalized with a consistent taking into account the temperature variation in transforming particles in the course of $P T$ and for media with internal variables. For these purposes a new approach based on a dissipation balance in a transforming material point is developed. A model problem concerning the nucleation of the thin infinite layer in a rigid-plastic half-space under prescribed normal and shear stresses is solved. The angle of inclination of the layer is determined based on a postulate of realizability [2] - [4]. Nontrivial effect of a shear stresses on the PT pressure is related with a necessity to meet plasticity condition in the parent phase.

\section{CONDITIONS OF NUCLEATION AND IN'TERFACE PROPAGATION}

Consider a volume $V$ of multiphase material with a boundary $S$ and prescribed boundary data on $S$. Assume that in small volume $V_{n} \in V$ with the boundary $\Sigma_{n}$ some PT occurs during the time $\Delta t$. We admit the second law of thermodynamic for each point of a volume $V_{n}$ in the form of the Plank inequality

$$
\mathcal{D}=\sigma: \dot{\varepsilon}-\rho \dot{\psi}-\rho s \dot{\theta} \geq 0 .
$$

Here $\mathcal{D}$ is the rate of dissipation per unit volume, $\rho$ the mass density, $s$ the entropy, $\psi$ the specific Helmholtz free energy, $\sigma$ and $\varepsilon$ the stress and strain tensors, $\theta$ the temperature. The total dissipation increment during the PT in each transforming material point is defined as

$$
N:=\int_{t}^{t+\Delta t} \mathcal{D} d t=\int_{\varepsilon_{1}}^{\varepsilon_{2}} \sigma: d \varepsilon-\rho\left(\psi_{2}-\psi_{1}\right)-\int_{\theta_{1}}^{\theta_{2}} \rho s d \theta .
$$

Assume that during PT three dissipative processes occur: PT itself, plastic flow and the process of variation of some unspecified internal variable $g$. As an example of internal variable a dislocation density tensor or tensor of internal stresses can be considered. The dissipation increment in the 
course of PT due to plastic flow and variation of internal variable can be represented in the following form

$$
N_{p g}=\int_{t}^{t+\Delta t}\left(X_{p}: \dot{\varepsilon}_{p}+X_{g}: \dot{g}\right) d t
$$

where $\boldsymbol{X}_{p}=\boldsymbol{\sigma}-\rho \frac{\partial \psi}{\partial \varepsilon_{p}}$ and $\boldsymbol{X}_{g}=-\rho \frac{\partial \psi}{\partial \boldsymbol{g}}$ are generalized dissipative forces, conjugated with plastic strain rate $\dot{\varepsilon}_{p}$ and $\dot{g}$ respectively. The expression for $X_{p}$ and $\boldsymbol{X}_{g}$ is derived using the standard thermodynamical procedure for materials without PT $[5,6]$. The dissipation increment due to PT itself $X$ is a difference between $N$ and $N_{p g}$, i.e.

$$
X=\int_{\varepsilon_{1}}^{\varepsilon_{2}} \sigma: d \varepsilon-\rho\left(\psi_{2}-\psi_{1}\right)-\int_{\theta_{1}}^{\theta_{2}} \rho s d \theta-\int_{t}^{t+\Delta t}\left(X_{p}: \dot{\varepsilon}_{p}+X_{g}: \dot{g}\right) d t
$$

The simplest assumption that all three dissipative processes are mutually independent results in conditions that dissipation increment due to each of dissipative processes should be nonnegative, in particular $X \geq 0$. Consequently, at $X<0 \mathrm{PT}$ is impossible. The condition $X=0$ is the criterion of PT without dissipation due to PT, because PT is possible (noncontradicts to second law of thermodynamics) and dissipation increment due to PT is zero.

Since practically all martensitic transformations, even in elastic materials, are accompanied with a dissipation and a hysteresis, the PT criterion has the following form

$$
X=k \text {. }
$$

Here $k$ is an experimentally determined value of dissipation due to PT, which can depend on parameters $\theta, \varepsilon_{p}, g, \ldots$; we shall designate them $y$. At $X<k$ PT is impossible.

For each point of nuclei $V_{n}$ PT criterion (5) should be met. Integrating this criterion over the volume $V_{n}$ we obtain the necessary condition of nucleation

$$
\int_{V_{n}} X d V_{n}=\int_{V_{n}} k d V_{n}
$$

or taking into account Eq. (4) for $X$

$$
\begin{aligned}
\int_{V_{n}} \int_{\varepsilon_{1}}^{\varepsilon_{2}} \boldsymbol{\sigma}: d \boldsymbol{\varepsilon} d V_{n} & =\int_{t}^{t+\Delta t} \int_{\Sigma_{n}} \boldsymbol{p} \cdot \boldsymbol{v} d \Sigma_{n} d t=\int_{V_{n}} \rho\left(\psi_{2}-\psi_{1}\right) d V_{n}+\int_{V_{n}} \int_{\theta_{1}}^{\theta_{2}} \rho s d \theta d V_{n}+ \\
& +\int_{V_{n}}^{t+\Delta t} \int_{t}^{t}\left(\boldsymbol{X}_{p}: \dot{\boldsymbol{\varepsilon}}_{p}+\boldsymbol{X}_{g}: \dot{\boldsymbol{g}}\right) d t d V_{n}+\int_{V_{n}} k d V_{n}
\end{aligned}
$$

where $v$ is the velocity on $\Sigma_{n}$ from the side of nucleus, $p=\sigma \cdot n$ the stress vector. Note that Gauss theorem was used.

Assume that the volume $V_{n}$ is obtained by interface $\Sigma$ propagation with a normal velocity $v_{n}$ during time $\Delta t$, i.e. it is bounded by surfaces $\Sigma_{t}$ and $\Sigma_{t+\Delta t}$ at time $t$ and $t+\Delta t$ respectively, as well as by two lateral infinitesimal surfaces with the heights $v_{n} \Delta t$. Let us transform the stress work integral in Eq. (7).

On the moving coherent interface the position vector and the traction vector $p$ are continuous, $p_{2}=p_{1}$, and due to compatibility condition $[1,4]$

$$
[\boldsymbol{F}]=-[\boldsymbol{v}] \boldsymbol{n} / v_{n}, \quad \text { whence } \quad[\boldsymbol{v}]=-[\boldsymbol{F}] \cdot \boldsymbol{n} v_{n} \quad \text { and } \quad[\boldsymbol{F}]=[\boldsymbol{F}] \cdot \boldsymbol{n} \boldsymbol{n} .
$$

Here $n$ is the unit normal to the interface, $\boldsymbol{F}=\varepsilon+\boldsymbol{\omega}, \boldsymbol{\omega}$ the rotation tensor, $[\boldsymbol{a}]:=\boldsymbol{a}_{2}-\boldsymbol{a}_{1}$. Then 
neglecting all the terms of order $\Delta t^{2}$ we obtain

$$
\begin{aligned}
\int_{t}^{t+\Delta t} \int_{\Sigma} p \cdot v d \Sigma d t & =\left(\int_{\Sigma_{t+\Delta t}} p_{1} \cdot v_{1} d \Sigma_{t+\Delta t}-\int_{\Sigma_{t}} p_{2} \cdot v_{2} d \Sigma_{t}\right) \Delta t=-\int_{\Sigma_{t}}[p \cdot v] \Delta t d \Sigma_{t}, \\
-[p \cdot v] & =-p \cdot[v]=n \cdot \sigma \cdot[F] \cdot n v_{n}=\sigma:[F] \cdot n n v_{n}=\sigma:[\varepsilon] v_{n},
\end{aligned}
$$

i.e. work-producing components of stress tensor are fixed in the course of PT. Substitution of Eq. (9) into Eq. (7) with account for $d V_{n}=v_{n} \Delta t d \Sigma_{t}$ results in

$$
\boldsymbol{n} \cdot \boldsymbol{\sigma} \cdot[\boldsymbol{F}] \cdot \boldsymbol{n}=\boldsymbol{\sigma}:[\varepsilon]=\rho[\psi]+\int_{\theta_{1}}^{\theta_{2}} \rho s d \theta+\int_{t}^{t+\Delta t}\left(\boldsymbol{X}_{p}: \dot{\varepsilon}_{p}+\boldsymbol{X}_{g}: \dot{\boldsymbol{g}}\right) d t+k .
$$

For the interface propagation it is necessary that Eq. (10) is met at time $t+\Delta t$ as well [2] - [4]

$$
\boldsymbol{\sigma}_{\Delta}:\left[\varepsilon_{\Delta}\right]=\rho\left[\psi_{\Delta}\right]+\int_{\theta_{1 \Delta}}^{\theta_{2} \Delta} \rho s d \theta+\int_{\varepsilon_{p 1 \Delta}}^{\varepsilon_{p 2 \Delta}} \boldsymbol{X}_{p}: d \varepsilon_{p}+\int_{\boldsymbol{g}_{1 \Delta}}^{\boldsymbol{g}_{2 \Delta}} \boldsymbol{X}_{g}: d \boldsymbol{g}+k_{\Delta} .
$$

Eq. (11) is a counterpart of consistency condition in plasticity theory, where subscript $\Delta$ denotes that parameter is determined at time $t+\Delta t$. For an infinitesimal $\Delta t$ Eqs. (10) and (11) can be transformed into

$$
\begin{array}{r}
\left(\dot{\boldsymbol{\sigma}}+\boldsymbol{n} \cdot \boldsymbol{\nabla} \boldsymbol{\sigma} v_{n}\right):[\boldsymbol{\varepsilon}]+\boldsymbol{\sigma}:\left([\dot{\boldsymbol{\varepsilon}}]+\boldsymbol{n} \cdot[\nabla \boldsymbol{\varepsilon}] v_{n}\right)-\left([\dot{\psi}]+\boldsymbol{n} \cdot[\nabla \psi] v_{n}\right)- \\
-\left([\rho s \dot{\theta}]+\boldsymbol{n} \cdot[\rho s \boldsymbol{\nabla} \theta] v_{n}\right)-\left(\left[\boldsymbol{X}_{p}: \dot{\boldsymbol{\varepsilon}}_{p}\right]+\left[\boldsymbol{X}_{p}:(\boldsymbol{n} \cdot \nabla \boldsymbol{g})\right] v_{n}\right)- \\
-\left(\left[\boldsymbol{X}_{\boldsymbol{g}}: \dot{\boldsymbol{g}}\right]+\left[\boldsymbol{X}_{g}:(\boldsymbol{n} \cdot \boldsymbol{\nabla} \boldsymbol{g})\right] v_{n}\right)=\frac{\partial k}{\partial u_{n}} v_{n}+\frac{\partial k}{\partial \boldsymbol{y}} \cdot\left([\dot{\boldsymbol{y}}]+\boldsymbol{n} \cdot[\nabla \boldsymbol{y}] v_{n}\right)
\end{array}
$$

where $u_{n}$ is an interface displacement, $\dot{u}_{n}=v_{n}$. We have taken into account that $\boldsymbol{a}_{\Delta}=\boldsymbol{a}+\left(\dot{\boldsymbol{a}}+\boldsymbol{n} \cdot \boldsymbol{\nabla} \boldsymbol{a} v_{n}\right) \Delta t$ for all parameters, equation

$$
\begin{aligned}
& \int_{\varepsilon_{p 1 \Delta}}^{\varepsilon_{p 2} \Delta} X_{p}: d \varepsilon_{p}=\int_{\varepsilon_{p 1}}^{\varepsilon_{p 2}} X_{p}: d \varepsilon_{p}+\int_{\varepsilon_{p 2}}^{\varepsilon_{p 2 \Delta}} X_{p}: d \varepsilon_{p}-\int_{\varepsilon_{p 1}}^{\varepsilon_{p 1} \Delta} X_{p}: d \varepsilon_{p}= \\
= & \int_{\varepsilon_{p 1}}^{\varepsilon_{p 2}} X_{p}: d \varepsilon_{p}+\boldsymbol{X}_{p 2}:\left(\dot{\varepsilon}_{p 2}+n \cdot \nabla \varepsilon_{p 2} v_{n}\right) \Delta t-\boldsymbol{X}_{p 1}:\left(\dot{\varepsilon}_{p 1}+n \cdot \nabla \varepsilon_{p 1} v_{n}\right) \Delta t
\end{aligned}
$$

and similar equations for all other parameters. Here $\nabla$ is the gradient operator, the term $\nabla a$ appears due to the fact that the tensor $\boldsymbol{a}_{\Delta}$ is determined on the $\Sigma_{\Delta}$ surface, i.e. at point $\boldsymbol{r}+v_{n} n \Delta t$ $(\boldsymbol{r} \in \Sigma(t))$.

Eqs. (7) and (10) - (12) generalize equations, postulated in [2] - [4], for nonisothermal processes and media with internal variables. Note that at transition from the material point to the finite volume or surface additional energy terms can appear and should be taken into account. E.g., a change of surface energy should be added to right side of Eqs. (7) and (10). Additional dissipation threshold $k_{\Sigma}$ due to intersection of interface with dislocations, point defects, grains and subgrains boundaries should be added to $k$ in Eq. (10).

Note, that Eqs. (7) and (10) are valid for noncoherent PT with small jump of displacement as well, since we are using the velocity vector $v$ from the side of nucleus, i.e. the rate of relative sliding along the interface $[\boldsymbol{v}]^{s}$ is excluded from consideration and $[\boldsymbol{v}]$ should always meet the compatibility 
condition. We can define the total jump of velocity across the interface $[\boldsymbol{v}]^{T}=[\boldsymbol{v}]+[\boldsymbol{v}]^{s}$ and can use for the definition of $[\boldsymbol{v}]^{s}$ equations and extremum principles derived in [4].

Temperature variation in the course of PT can be determined using the first law of thermodynamics or entropy balance equation, in particularly, under assumption that process is adiabatic $[5,6]$.

\section{MODEL PROBLEM}

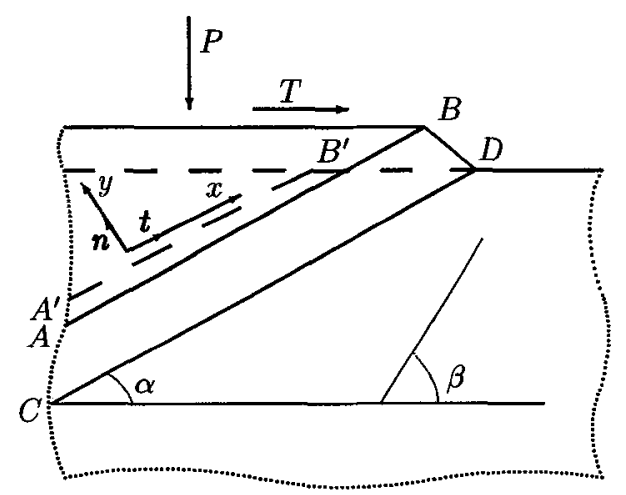

Figure 1: Coherent PT in tnin layer $A B C D$

Let us consider an infinite rigid-plastic half-space with prescribed normal $P$ and shear $T$ stresses on the whole surface (Fig. 1). Assume that a coherent PT transforms the thin infinite layer $A^{\prime} B^{\prime} D C$ in $A B D C$, which is inclined at some angle $\alpha$ to surface $S$. We shall consider a plane strain problem and assume that stress-strained field are homogeneous in the layer and stress field is fixed in the course of PT. Material outside the layer is rigid.

Similar problem at fixed $\alpha=0$ was solved in [3]. In [5] the temperature variation in the course of $\mathrm{PT}$ was taken into account. Here we shall define an "optimal" angle $\alpha$ and find limitation related with a fulfillment of the yield conditions outside the layer. Temperature variation due to the lack of place will be neglected.

In our case $\varepsilon=\varepsilon_{p}+\varepsilon_{t}$, where $\varepsilon_{t}$ is a transformation strain, $\dot{\boldsymbol{g}} \equiv 0$ and $\boldsymbol{X}_{p}=\boldsymbol{\sigma}$. In the local $x-y$ coordinate system we assume

$$
\boldsymbol{\varepsilon}_{t}=\left(0.5 \varepsilon_{0} i+\gamma^{t}(t n)_{s}\right) \xi,
$$

where $\xi$ is a parameter, which varies from 0 till 1 in the course of PT, $\varepsilon_{0}$ and $\gamma^{t}$ are the volumetric and shear transformation strains, $i$ the two dimensional unit tensor, subscript $s$ means symmetrisation. In this case PT criterion (7) results in

$$
X_{n}=0.5 \varepsilon_{o}\left(\sigma_{\alpha n}+\sigma_{\alpha t}\right)+\tau_{\alpha} \gamma^{t}-\Delta \psi^{\theta}=k(\alpha),
$$

where function $k(\alpha)$ characterizes an anisotropy of dissipative threshold; $\Delta \psi^{\theta}=\psi_{2}^{\theta}-\psi_{1}^{\theta}$ is the difference of thermal parts of $\psi$, which is a known function of temperature; $\sigma_{\alpha n}, \sigma_{\alpha t}$ and $\tau_{\alpha}$ are the normal, tangential and shear stresses in the layer, subscript $\alpha$ means that the stresses are defined in a local coordinate system $x-y$, inclined at the angle $\alpha$.

Let a slip line in the layer is inclined at an angle $\beta$ respective the surface $S$ (Fig 1). Per definition for a slip line is valid $\tau_{\beta}=0.5 \sigma_{y}$, where $\sigma_{y}$ is the yield limit.

We shall use known relations based on Moohr's circle

$$
\begin{array}{cc}
\sigma_{\alpha n}=\sigma \pm 0.5 \sigma_{y} \sin 2(\beta-\alpha) ; & \sigma_{\alpha t}=\sigma \mp 0.5 \sigma_{y} \sin 2(\beta-\alpha) ; \\
\tau_{\alpha}=0.5 \sigma_{y} \cos 2(\beta-\alpha) \quad \text { and } & \sigma:=0.5\left(\sigma_{\alpha n}+\sigma_{\alpha t}\right)=0.5\left(\sigma_{1}+\sigma_{2}\right),
\end{array}
$$

where $\sigma_{1}$ and $\sigma_{2}$ are the main stresses. The signs + or - in Eq. (16) should be chosen, based on knowledge, which inequality $\sigma_{\alpha n}>\sigma_{\alpha t}$ or $\sigma_{\alpha n}<\sigma_{\alpha t}$ is valid for the problem under consideration. The stresses (16), (17) meet automatically the Mises yield condition $\left(\sigma_{\alpha n}-\sigma_{\alpha t}\right)^{2}+4 \tau_{\alpha}^{2}=\sigma_{y}^{2}$, as well as a condition $\tau_{\alpha}=0.5 \sigma_{y}$, when $\alpha=\beta$. The line $B D$ remains horizontal for small displacement approximation. Corresponding to the boundary conditions on the line $B D$

$$
\text { for } \quad \alpha=0 \quad \text { is valid } \quad \sigma_{\alpha n}=P>\sigma_{\alpha t}, \quad \tau_{\alpha}=T .
$$

Consequently, $0.5 \sigma_{y} \cos 2 \beta=T ; \quad \sigma+0.5 \sigma_{y} \sin 2 \beta=P$, whence 


$$
\cos 2 \beta=\frac{2 T}{\sigma_{y}} \quad \text { and } \quad \sigma=P-0.5 \sigma_{y} \sqrt{1-\frac{4 T^{2}}{\sigma_{y}^{2}}} .
$$

Eq. $(19)_{1}$ determines an orientation of two orthogonal slip lines. Substitution of Eq. (17) $)_{2}$ in Eq. (15) gives

$$
X_{n}=\sigma \varepsilon_{o}+\tau_{\alpha} \gamma^{t}-\Delta \psi^{\theta}=k(\alpha)
$$

From a postulate of realizability [2] - [4] it follows

$$
X_{n}(\alpha)-k(\alpha) \rightarrow \max , \quad \text { whence } \quad \sin 2(\beta-\alpha)=\frac{k^{\prime}}{\sigma_{y} \gamma^{t}}
$$

is valid with $k^{\prime}:=\frac{\partial k}{\partial \alpha}$. Eq. (21) allows us to define the function $\alpha=q(\beta)$. Substituting $\sigma$ from Eq. (19) $)_{2}$ and $\alpha=q(\beta)$ in Eq. (20) we obtain the PT criterion

$$
\begin{gathered}
X_{n}=P \varepsilon_{o}-0.5 \varepsilon_{o} \sqrt{\sigma_{y 2}^{2}-4 T^{2}}+0.5 \sigma_{y 2} \gamma^{t} \cos 2(\beta-q(\beta))-\Delta \psi^{\theta}=k(q(\beta)), \\
\text { or } \quad(P-A)^{2}+T^{2}=0.25 \sigma_{y 2}^{2}, \quad P \geq A, \quad \text { where } \\
A:=\frac{\Delta \psi^{\theta}+k(q(\beta))-0.5 \sigma_{y 2} \gamma^{t} \cos 2(\beta-q(\beta))}{\varepsilon_{o}},
\end{gathered}
$$

which represents in a $P-T$ plane a half sphere with a radius $0.5 \sigma_{y 2}$, shifted on vector $A$ along the axis $P$ (Fig. 2). We took into account that $\sigma_{y}=\sigma_{y 2}$ (for the second phase). If $k^{\prime} \equiv 0$, then $\alpha=\beta$ (the layer coincides with a slip line) and

$$
A=\frac{\Delta \psi^{\theta}+k-0.5 \sigma_{y 2} \gamma^{t}}{\varepsilon_{o}}
$$

We should take also into account the inequality

$$
P^{2}+4 T^{2} \leq \sigma_{y 1}^{2}
$$

which means that applied stresses cannot violate the yield condition for the first phase and a material outside the layer is rigid. In a $P-T$ plane Eq. (26) represents an ellipsis with semiaxis $\sigma_{y 1}$ and $0.5 \sigma_{y 1}$. Consequently, the PT - criterion represents in a $P-T$ plane the part of a half sphere (23) lying inside the ellipsis (26) (Fig. 2). Let us analyze the obtained results.

If the restriction (26) should not be taken into account, then the increase of $T$ decreases the PT pressure, and maximal decrease is equal to $0.5 \sigma_{y_{2}}$ at $T=0.5 \sigma_{y_{2}}$. Inequality (26) results in a several important limitations (see Fig. 2):

1. PT is possible at $-\sigma_{y 1} \leq A+0.5 \sigma_{y 2} \leq \sigma_{y 1}$.

2. The increase of $T$ decreases the PT pressure when the sphere (23) lies inside the ellipsis (26). An additional increase of $T$ makes $\mathrm{PT}$ impossible. In particularly at $\sigma_{y_{2}} \geq \sigma_{y 1}$ and $A=\sigma_{y 1}-0.5 \sigma_{y 2}$ or $A=-\sigma_{y_{1}}-0.5 \sigma_{y_{2}} \mathrm{PT}$ is possible at $T \equiv 0$ only (points $P= \pm \sigma_{y 1}$ ).

3. The larger $\frac{\sigma_{y 1}}{\sigma_{y 2}}$, the contribution of $T$ is more significant. For $\sigma_{y 1} \leq 0.5 \sigma_{y 2} \quad T$ practically does not affect the $P$.

A comparison of Eq. (22) for the "optimal" angle $\alpha$ with the results obtained in [3] for $\alpha=0$ shows that:

1. The term $\sqrt{\sigma_{y}^{2}-4 T^{2}}$ is independent of $\alpha$;

2. The term $\frac{\tau \gamma^{t}}{\varepsilon_{0}}$ (for $\alpha=0$ ) reaches for the "optimal" angle $\alpha$ its maximal value $\frac{0.5 \sigma_{y} \gamma^{t}}{\varepsilon_{0}}$. 


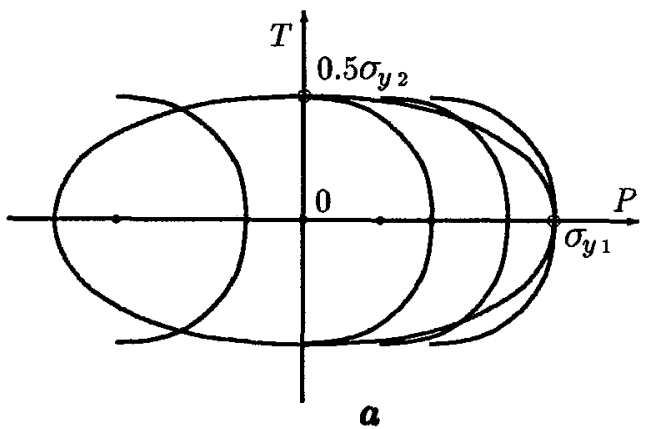

a

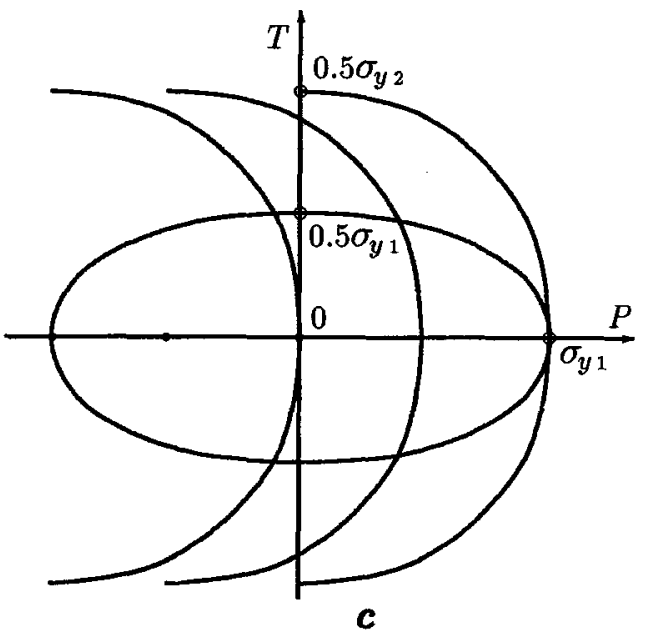

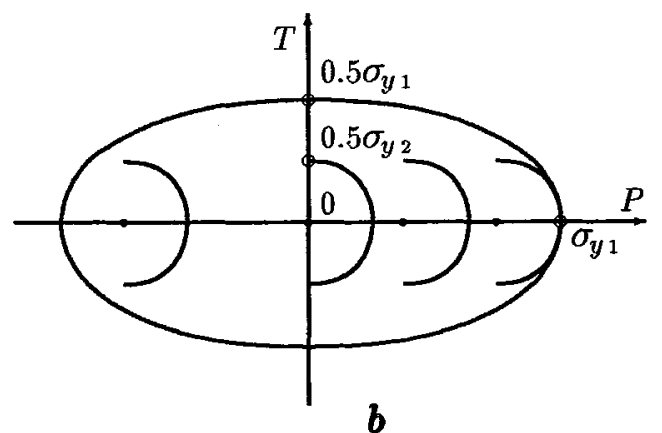

Figure 2: PT criterion (half sphere with a radius $0.5 \sigma_{y_{2}}$ and a center in a point $A$ ) and yield condition (an ellipsis):

a) $\sigma_{y_{1}}=\sigma_{y_{2}}$

b) $\sigma_{y_{1}}=2 \sigma_{y_{2}}$;

c) $\sigma_{y_{1}}=0.5 \sigma_{y_{2}}$;

\section{CONCLUDING REMARKS}

The derived nucleation and interface propagation conditions are rather general and independent of the materials constitutive equations. At the next step the simplified models of transforming volume should be developed, e.g. macroscopically uniformly [2] or with constant strain gradient deformed volume, with account for inertia effects, with a wave mechanism of nucleus growth and so on.

\section{Acknowledgments}

The financial support of Alexander von Humboldt Foundation and Volkswagen Foundation, as well as discussions with Prof. Erwin Stein are gratefully acknowledged.

\section{References}

1 ] Fischer F.D., Berveiller M., Tanaka K., Oberaigner E., Arch. Appl. Mech. 64 (1994) 54-85.

2 Levitas V. I., Mech. Res. Commun. 22 (1995) 87-94.

3 ] Levitas V. I., Journal de Physique IV, Colloque C2 5 (1995) 41-46.

4 ] Levitas V. I., Int. J. Eng. Sci., Parts 1 and 2, 33 (1995) 921-971.

5 ] Levitas V.I., Mech. Res. Commun. (1995) (submitted).

6 ] Levitas V.I., Proc. MECAMAT-95 (in press).

[ 7 ] Levitas V.I., Thermodynamische Phasenumwandlungstheorie und eine Ähnlichkeit zur Plastizitätstheorie. Uni. Hannover, IBNM - Bericht 95/1 (1995) 45 p. 